\title{
Inventory of Chondrichthyes and Actinopterygii species collected in the central coast of São Paulo State, Brazil
}

\author{
Maria Luiza Flaquer da Rocha ${ }^{1}$ \& June Ferraz Dias ${ }^{1,2}$ \\ ${ }^{1}$ Universidade de São Paulo, Instituto Oceanográfico, Departamento de Oceanografia Biológica, \\ São Paulo, SP, Brazil. \\ ${ }^{2}$ Corresponding author: June Ferraz Dias, e-mail: junedias@usp.br
}

\begin{abstract}
ROCHA, M.L.F., DIAS, J.F. Inventory of Chondrichthyes and Actinopterygii species collected in the central coast of São Paulo State, Brazil. Biota Neotropica. 15(2): e20140136. http://dx.doi.org/10.1590/ 1676-06032015013614
\end{abstract}

\begin{abstract}
This biodiversity inventory of the central coast of São Paulo State presents species of demersal and pelagic fishes of Santos Bay, Bertioga Channel, and adjacent continental shelf, between São Sebastião and Peruíbe, from the coast till a depth of $100 \mathrm{~m}$. Samples were taken during oceanographic campaigns using otter trawls, between November 2004 and February 2006. A total of 56,095 individuals were collected, belonging to the classes Chondrichthyes (three orders and fourteen species) and Actinopterygii (fifteen orders and 141 species). This list adds 27 species to those explicitly cited for the coast of São Paulo state, contributing to the knowledge of this group.
\end{abstract}

Keywords: Ichthyofauna, Santos Bay, Bertioga Channel, Continental Shelf, Southeastern Brazilian Coast.

ROCHA, M.L.F., DIAS, J.F. Inventário das espécies de Chondrichthyes e Actinopterygii coletadas no litoral central do estado de São Paulo, Brasil. Biota Neotropica. 15(2): e20140136. http://dx.doi.org/10. 1590/1676-06032015013614

Resumo: Este inventário comentado da biodiversidade do litoral central do estado de São Paulo apresenta as espécies de peixes demersais e pelágicas da baía de Santos, do canal de Bertioga e da plataforma continental adjacente, entre Peruíbe e São Sebastião, da costa até $100 \mathrm{~m}$ de profundidade. Os exemplares foram capturados durante campanhas oceanográficas, utilizando-se redes de arrasto de fundo com portas, entre novembro de 2004 e fevereiro de 2006. Foram capturados um total de 56.095 indivíduos pertencentes às Classes Chondrichthyes (três ordens e quatorze espécies) e Actinopterygii (quinze ordens e 141 espécies). Esta lista amplia em 27 espécies as explicitamente citadas para o litoral do estado de São Paulo, contribuindo para o conhecimento do grupo.

Palavras-chave: Ictiofauna, baía de Santos, canal de Bertioga, plataforma continental adjacente, costa sudeste brasileira.

\section{Introduction}

Species lists are important tools in biodiversity and fish community structure studies, not only to evaluate current and past ichthyofauna records and richness but also as a reference document to environmental agencies and consultancies. Considering these different purposes and the poor environmental quality of the coastal areas, fish have also been used to assess the ecological status of marine environments (Henriques et al. 2008).

Some ichthyofaunistic surveys have been conducted in the central coast of São Paulo state; among these surveys, we can highlight Vazzoler (1970), Paiva-Filho et al. (1987), Giannini \& Paiva-Filho (1990), and Graça-Lopes et al. (1993) for the Santos Bay and Barbanti et al. (2013) for the Bertioga Channel. Although already investigated, little information has been published about the ichthyofauna inventory of the continental shelf ecosystem (Vazzoler et al. 1982, Fachinni, 1999). However, this lack of information is not restricted to the coast of São Paulo. Most of ichthyofauna information is found in academic products (Dissertations and Theses). One of the major surveys was the "Program of Evaluation of the Sustainable Potential for Living Resources in the Exclusive Economic Zone (Programa de Avaliação do Potencial Sustentável de Recursos Vivos na Zona Econômica Exclusiva - REVIZEE)",

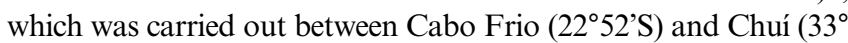
$41^{\prime}$ S) and which resulted in a series of technical documents and available information; however, this campaigns occurred in deeper waters than those examined in the present study, between 100 and 1,000 meters.

Menezes (2011) published a checklist of marine fishes for the state of São Paulo, which was based on the Catalog of Marine Fish Species of Brasil that he and colleagues published in 2003 .

The purpose of this article is to present an updated species list, based on the current knowledge of the biodiversity fish in the central coast of São Paulo state. 

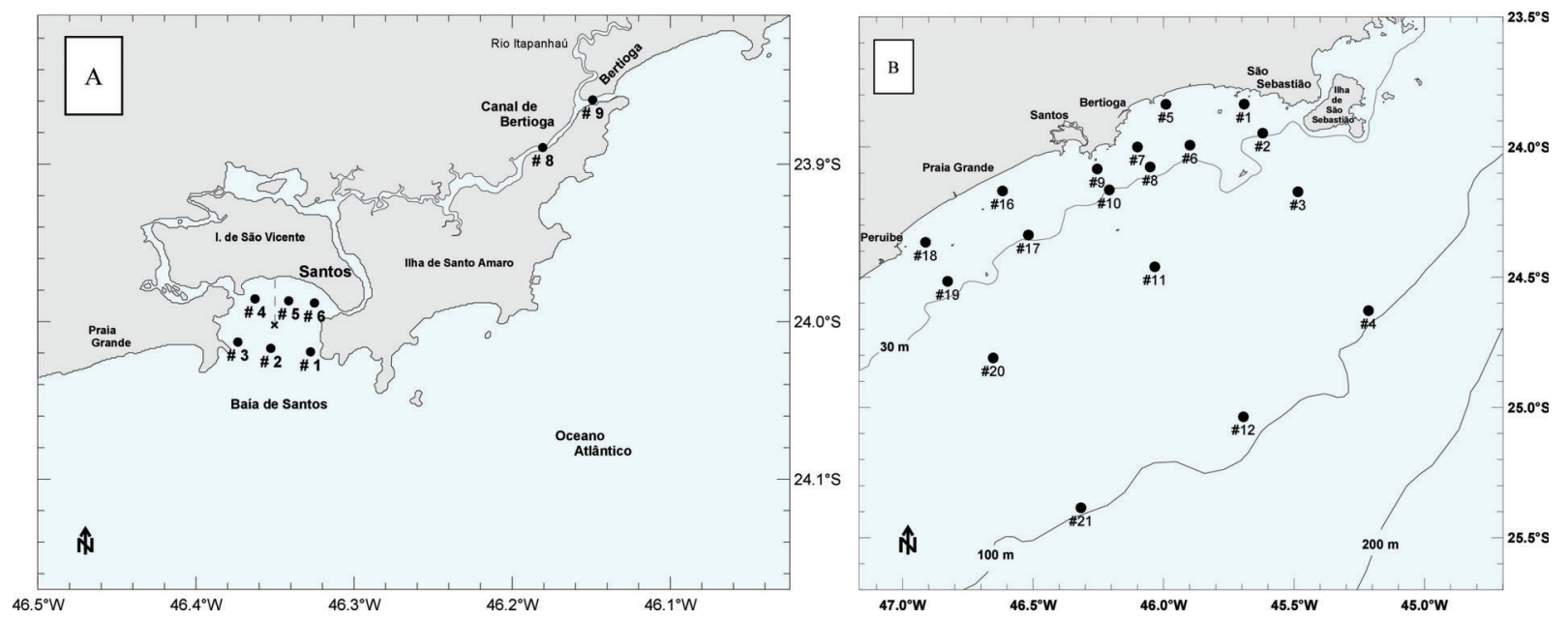

Figure 1. Maps of Santos Bay, Bertioga Channel (A) and adjacent Continental Shelf in the central coast of the state of São Paulo (B), with collection stations of the ichthyofauna.

\section{Material and Methods}

The sampled areas are part of the Santos-São Vicente estuarine system, including the adjacent continental shelf (Fig. 1). The Santos Bay $\left(23.98^{\circ}-24.04^{\circ} \mathrm{S} ; 46.4^{\circ}-46.3^{\circ} \mathrm{W}\right)$ is a semi-sheltered bay, with depths ranging from 5 to $15 \mathrm{~m}$. It is bordered by beaches and two natural channels (Santos and São Vicente) to the north and by the Atlantic Ocean to the south. It has an outfall sewer. The sediment type from Santos Bay was classified as very fine sand at the western side and clay and silt in the central-eastern area.

The Bertioga Channel is located at the eastern end of the Santos region $\left(23.85-23.60^{\circ} \mathrm{S} ; 46.18-46.25^{\circ} \mathrm{W}\right)$ and is considered a secondary connection to the ocean of the estuarine system of Santos - São Vicente. The channel is $25 \mathrm{~km}$ long with an average depth between 3 and $6 \mathrm{~m}$ and width ranging from 200 to $700 \mathrm{~m}$. The sediment types were classified as clay and medium silt along the channel and fine sand on both borders.

On the continental shelf adjacent to the estuarine system, the sampling area was concentrated between São Sebastião $\left(23^{\circ} 45^{\circ} \mathrm{S}\right)$ and Peruíbe $\left(24^{\circ} 19^{\circ} \mathrm{S}\right)$ at depths shallower than $100 \mathrm{~m}$. In this region, the sediment types were classified as sand for all coastal areas up to $80 \mathrm{~m}$, a transition from sandy silt to silt-clay type sediment occurred between 80 and $100 \mathrm{~m}$.

In the Santos Bay, specimens were caught monthly at six oceanographic stations, between November 2004 and December 2005 (except for the month of December 2004), three along the entrance of the bay and three along the beaches (stations 1 to 6 ; Fig. 1A). In the internal stations, the local depth varied between 5.7 and $9.9 \mathrm{~m}$, while it varied between 10 and $14.3 \mathrm{~m}$ in the external stations. In the Bertioga Channel, samples were collected monthly from September to December 2005 in two channel points (stations 8 and 9; Fig. 1A), one more internal (between the bar and Largo do Candinho) and another more external point close to channel north mouth. An otter-trawl was used to collect fish in these areas, with a mesh size of 40 $\mathrm{mm}$ in the arms and $30 \mathrm{~mm}$ in the bagger; the otter-trawl was $11 \mathrm{~m}$ long. The effort unit was a 10-min tow at a speed of two knots.

Two expeditions were made at the continental shelf, one in the winter of 2005 (August/September) and another in the summer of 2006 (February), totaling 38 oceanographic stations at depths varying between 14 and $94 \mathrm{~m}$ (Fig. 1B). An ottertrawl with $17 \mathrm{~m}$ length, and a mesh size of $60 \mathrm{~mm}$ in the arms and $25 \mathrm{~mm}$ in the bagger was used. The effort unit was a 30-minute tow at an average speed of two knots.

The caught specimens were sacrificed by cooling, sorted and identified based on Figueiredo \& Menezes (1978, 1980, 2000), Menezes \& Figueiredo (1980, 1985) manuals; Marceniuk (2005) and Gomes et al. (2010) identification keys, and additional taxonomic information of Nelson (2006). Data of total length and standard length (in $\mathrm{mm}$ ) were taken for each specimen. After identification and data recording on board, all Chondrichthyes specimens were immediately released to the sea while still alive.

The Menezes et al. (2003) catalog, the Websites Catalog of Fishes (CAS), FishBase, Advanced Search Report (ITIS), and World Register of Marine Species (WorMS); and Marceniuk \& Menezes (2007) and Menezes et al. (2015) publications were utilized to update the nomenclature. The testimony specimens are frozen at ECORREP (Reproductive Ecology and Recruitment Laboratory - Oceanographic Institute-University of São Paulo) and will be added to the ColBIO (Coleções Biológicas Prof. Edmundo Ferraz Nonato-IOUSP).

\section{Results}

The collected species are presented in phylogenetic order according to Menezes et al. (2003), and the lists are separated per area. The minimal and maximal lengths per species are also shown. If the caudal fin was damaged or absent, the standard length of the species was provided.

\section{Santos Bay}

The Santos Bay was represented by 94 species of Actinopterygii and by only one species of Chondrichthyes (Rajiformes) (Table 1).

\section{Bertioga Channel}

The Bertioga Channel contained 50 species, all belonging to Actinopterygii (Table 2). 
Ichthyofauna of the central coast of São Paulo

Table 1. List of species collected at Santos Bay between November 2004 and December 2005, with respective total maximum (TL max) and minimum (TL min) lengths in millimeters. In case of a single specimen, its length is shown at "TL max" column. SL $=$ standard length.

\begin{tabular}{|c|c|c|c|c|}
\hline Order & Family & Species & TL min & TL max \\
\hline Rajiformes & Rhinobatidae & Rhinobatos percellens (Walbaum, 1792) & 235 & 540 \\
\hline Anguilliformes & Ophichthidae & Ophichthus gomesii (Castelnau, 1855) & 503 & 600 \\
\hline \multirow{14}{*}{ Clupeiformes } & Engraulidae & Anchoa filifera (Fowler, 1915) & 72 & 105 \\
\hline & & Anchoa januaria (Steindachner, 1879) & 65 & 92 \\
\hline & & Anchoa marinii Hildebrand, 1943 & 64 & 95 \\
\hline & & Anchoa spinifer (Valenciennes, 1848) & 60 & 180 \\
\hline & & Anchoa tricolor (Spix \& Agassiz, 1829) & & 69 \\
\hline & & Anchovia clupeoides (Swainson, 1839) & & 66 \\
\hline & & Anchoviella brevirostris (Gunther, 1868) & & 49 \\
\hline & & Anchoviella lepidentostole (Fowler, 1911) & 52 & 165 \\
\hline & & Lycengraulis grossidens (Agassiz, 1829) & 60 & 135 \\
\hline & Pristigasteridae & Chirocentrodon bleekerianus, (Poey, 1867) & 56 & 61 \\
\hline & & Pellona harroweri (Fowler, 1917) & 24 & 142 \\
\hline & Clupeidae & Harengula clupeola (Cuvier, 1829) & 67 & 187 \\
\hline & & Opisthonema oglinum (Lesueur, 1818) & 80 & 95 \\
\hline & & Platanichthys platana (Regan, 1917) & & 63 \\
\hline \multirow[t]{6}{*}{ Siluriformes } & Ariidae & Aspistor luniscutis (Valenciennes, 1840) & 60 & 335 \\
\hline & & Bagre bagre (Linnaeus, 1766) & 65 & 246 \\
\hline & & Cathorops spixii (Agassiz, 1829) & 41 & 366 \\
\hline & & Genidens barbus (Lacepède, 1803) & 55 & 255 \\
\hline & & Genidens genidens (Cuvier, 1829) & 171 & 313 \\
\hline & & Notarius grandicassis (Valenciennes, 1840) & 70 & 139 \\
\hline Aulopiformes & Synodontidae & Synodus foetens (Linnaeus, 1766) & 45 & 203 \\
\hline Gadiformes & Phycidae & Urophycis brasiliensis (Kaup, 1858) & & 131 \\
\hline Batrachoidiformes & Batrachoididae & Porichthys porosissimus (Cuvier, 1829) & 32 & 237 \\
\hline Lophiiformes & Ogcocephalidae & Ogcocephalus vespertilio (Linnaeus, 1758) & & 67 \\
\hline \multirow[t]{3}{*}{ Scorpaeniformes } & Scorpaenidae & Scorpaena brasiliensis Cuvier, 1829 & & 206 \\
\hline & Triglidae & Bellator brachychir (Regan, 1914) & & 91 \\
\hline & & Prionotus punctatus (Bloch, 1793) & 35 & 160 \\
\hline \multirow[t]{27}{*}{ Perciformes } & Centropomidae & Centropomus parallelus Poey, 1860 & 243 & 540 \\
\hline & Serranidae & Diplectrum radiale (Quoy \& Gaimard, 1824) & 107 & 218 \\
\hline & & Rypticus randalli Courtenay, 1967 & 103 & 204 \\
\hline & & Rypticus sp. Cuvier \& Valenciennes, 1829 & & 128 \\
\hline & Carangidae & Chloroscombrus chrysurus (Linnaeus, 1766) & 37 & 105 \\
\hline & & Hemicaranx amblyrhynchus (Cuvier, 1833) & 77 & 115 \\
\hline & & Oligoplites saliens (Bloch, 1793) & 119 & 167 \\
\hline & & Selene setapinnis (Mitchill, 1815) & 3 & 235 \\
\hline & & Selene vomer (Linnaeus, 1758) & 27 & 152 \\
\hline & & Trachinotus carolinus (Linnaeus, 1766) & 51 & 201 \\
\hline & Gerreidae & Diapterus auratus Ranzani, 1842 & 119 & 130 \\
\hline & & Diapterus rhombeus (Cuvier, 1829) & 100 & 198 \\
\hline & & Eucinostomus argenteus Baird \& Girard, 1855 & 100 & 141 \\
\hline & & Eucinostomus gula (Quoy \& Gaimard, 1824) & 104 & 180 \\
\hline & & Eucinostomus melanopterus (Bleeker, 1863) & 122 & 194 \\
\hline & Haemulidae & Conodon nobilis (Linnaeus, 1758) & 70 & 182 \\
\hline & & Genyatremus luteus (Bloch, 1790) & 90 & 194 \\
\hline & & Orthopristis ruber (Cuvier, 1830) & 140 & 302 \\
\hline & & Pomadasys corvinaeformis (Steindachner, 1868) & 64 & 102 \\
\hline & Polynemidae & Polydactylus oligodon (Gunther, 1860) & 164 & 167 \\
\hline & & Polydactylus virginicus (Linnaeus, 1758) & & 156 \\
\hline & Sciaenidae & Bairdiella ronchus (Cuvier, 1830) & 61 & 261 \\
\hline & & Ctenosciaena gracilicirrhus (Metzelaar, 1919) & 89 & 107 \\
\hline & & Cynoscion jamaicensis (Vaillant \& Bocourt, 1883) & 39 & 164 \\
\hline & & Cynoscion leiarchus (Cuvier, 1830) & 40 & 68 \\
\hline & & Cynoscion sp. Gill, 1861 & 25 & 51 \\
\hline & & Cynoscion virescens (Cuvier, 1830) & 66 & 331 \\
\hline
\end{tabular}


Table 1. Continued.

\begin{tabular}{|c|c|c|c|c|}
\hline Order & Family & Species & TL min & TL max \\
\hline & & Isopisthus parvipinnis (Cuvier, 1830) & 20 & 181 \\
\hline & & Larimus breviceps Cuvier, 1830 & 47 & 222 \\
\hline & & Macrodon atricauda (Gunther, 1880) & 22 & 355 \\
\hline & & Menticirrhus americanus (Linnaeus, 1758) & 63 & 410 \\
\hline & & Menticirrhus littoralis (Holbrook, 1847) & 80 & 142 \\
\hline & & Micropogonias furnieri (Desmarest, 1823) & 40 & 478 \\
\hline & & Nebris microps Cuvier, 1830 & 35 & 290 \\
\hline & & Ophioscion punctatissimus Meek \& Hildebrand, 1925 & 99 & 144 \\
\hline & & Paralonchurus brasiliensis (Steindachner, 1875) & 44 & 240 \\
\hline & & Stellifer brasiliensis (Shultz, 1945) & 36 & 222 \\
\hline & & Stellifer rastrifer (Jordan, 1889) & 32 & 220 \\
\hline & & Stellifer sp. Oken, 1817 & 40 & 137 \\
\hline & & Stellifer stellifer (Bloch, 1790) & 42 & 201 \\
\hline & & Umbrina canosai Berg, 1895 & 88 & 151 \\
\hline & & Umbrina coroides Cuvier, 1830 & 125 & 151 \\
\hline & Ephippidae & Chaetodipterus faber (Broussonet, 1782) & 52 & 233 \\
\hline & Stromateidae & Peprilus paru (Linnaeus, 1758) & 29 & 125 \\
\hline \multirow[t]{12}{*}{ Pleuronectiformes } & Paralichthyidae & Citharichthys dinoceros Goode \& Bean, 1886 & & 139 \\
\hline & & Citharichthys macrops Dresel, 1885 & & 116 \\
\hline & & Citharichthys spilopterus Gunther, 1862 & 83 & 130 \\
\hline & & Etropus crossotus Jordan \& Gilbert, 1882 & 63 & 118 \\
\hline & & Etropus longimanus Norman, 1933 & & 93 \\
\hline & Achiridae & Achirus declivis Chabanaud, 1940 & 81 & 143 \\
\hline & & Achirus lineatus (Linnaeus, 1758) & 64 & 139 \\
\hline & & Catathyridium garmani (Jordan, 1889) & & 62 \\
\hline & & Trinectes paulistanus (Miranda Ribeiro, 1915) & 58 & 205 \\
\hline & Cynoglossidae & Symphurus jenynsi Evermann \& Kendall, 1906 & & SL 120 \\
\hline & & Symphurus tessellatus (Quoy \& Gaimard, 1824) & 61 & 177 \\
\hline & & Symphurus trewavasae Chabanaud, 1948 & 123 & 147 \\
\hline \multirow[t]{7}{*}{ Tetraodontiformes } & Monacanthidae & Monacanthus ciliatus (Mitchill, 1818) & & 26 \\
\hline & & Stephanolepis hispidus (Linnaeus, 1766) & 24 & 25 \\
\hline & Tetraodontidae & Lagocephalus laevigatus (Linnaeus, 1766) & 34 & 115 \\
\hline & & Sphoeroides greeleyi Gilbert, 1900 & 35 & 177 \\
\hline & & Sphoeroides spengleri (Bloch, 1785) & & 90 \\
\hline & & Sphoeroides testudineus (Linnaeus, 1758) & 43 & 260 \\
\hline & Diodontidae & Chilomycterus spinosus (Linnaeus. 1758) & 56 & 162 \\
\hline
\end{tabular}

\section{Continental shelf}

One hundred fifteen species occurred on the continental shelf, 14 of which belong to Chondrichthyes (Squaliformes, Squatiniformes, and Rajiformes Orders), and the remainder belong to Actinopterygii (Table 3).

\section{Discussion}

Menezes (2011) refers to 594 species of marine fish in the coast of São Paulo state based on the "Catalog of Marine Fish Species of Brazil". In the present study, 154 species were caught in the central coast, which represents approximately $26 \%$ of the total evaluated species. This number is expressive when considering that the investigated areas were restricted to soft bottoms and that a single fishing gear was utilized. In general, the restriction to depths lower than $100 \mathrm{~m}$ eliminates mesopelagic fish, such as Myctophidae, Stomiidae, and Sternoptychidae; the oceanic Xiphidae, Scombridae, Gempylidae, and Echeneidae; the larger Carangidae, and many
Elasmobranchii species. The species associated with rocky or coralline sea bottoms, such as Serranidae, Haemulidae, Labridae, Labrisomidae, Pomacanthidae, Pomacentridae, Scorpaenidae, and Scaridae; and those of very shallow areas, such as Gobiidae and Blenniidae, also were not caught. Moreover, the stations were widely spaced in the case of continental shelf, and only two sampling seasons were utilized.

Despite the above restrictions and considering the three sampled areas, this inventory adds 27 species to the checklist published by Menezes (2011), which reports the ichthyofauna of all coastal areas in São Paulo state (Tab. 4).

Comparing the current inventory, in which 95 species are recorded for the Santos Bay, with the list of species collected by the end of the 1980s in the same area (Ribeiro Neto, 1989) some important differences may be noticed. Some groups, such as Serranidae, Haemulidae, and top predators like rays, morays, barracudas, and common snooks, were not captured. Conversely, ten different species of soles and two species of puffers appear in this inventory and were not part of the fauna 25 years ago. Despite the differences and environmental degradation, 
Table 2. List of species collected at Bertioga Channel between September and December 2005, with respective total maximum (TL max) and minimum (TL min) lengths in millimeters. In case of a single specimen, its length is shown at "TL max" column.

\begin{tabular}{|c|c|c|c|c|}
\hline Order & Family & Species & TL min & TL max \\
\hline \multirow[t]{7}{*}{ Clupeiformes } & Engraulidae & Anchoa januaria (Steindachner, 1879) & & 77 \\
\hline & & Anchoviella lepidentostole (Fowler, 1911) & 70 & 180 \\
\hline & & Cetengraulis edentulus (Cuvier, 1829) & 137 & 182 \\
\hline & & Lycengraulis grossidens (Agassiz, 1829) & 160 & 246 \\
\hline & Pristigasteridae & Pellona harroweri (Fowler, 1917) & 54 & 134 \\
\hline & Clupeidae & Harengula clupeola (Cuvier, 1829) & 56 & 187 \\
\hline & & Opisthonema oglinum (Lesueur, 1818) & 77 & 102 \\
\hline \multirow[t]{4}{*}{ Siluriformes } & Ariidae & Aspistor luniscutis (Valenciennes 1840) & 109 & 370 \\
\hline & & Cathorops spixii (Agassiz, 1829) & 80 & 240 \\
\hline & & Genidens barbus (Lacepède, 1803) & 113 & 289 \\
\hline & & Genidens genidens (Cuvier, 1829) & 110 & 294 \\
\hline Aulopiformes & Synodontidae & Synodus foetens (Linnaeus, 1766) & & 190 \\
\hline Mugiliformes & Mugilidae & Mugil curvidens Valenciennes 1836 & & 222 \\
\hline Scorpaeniformes & Triglidae & Prionotus punctatus (Bloch, 1793) & 43 & 141 \\
\hline \multirow[t]{21}{*}{ Perciformes } & Centropomidae & Centropomus parallelus Poey, 1860 & 185 & 266 \\
\hline & & Centropomus undecimalis (Bloch, 1792) & 810 & 1080 \\
\hline & Serranidae & Diplectrum radiale (Quoy \& Gaimard, 1824) & 81 & 190 \\
\hline & & Rypticus sp. Cuvier \& Valenciennes, 1829 & & 132 \\
\hline & Carangidae & Chloroscombrus chrysurus (Linnaeus, 1766) & 49 & 100 \\
\hline & & Selene vomer (Linnaeus, 1758) & 44 & 478 \\
\hline & Gerreidae & Diapterus auratus Ranzani, 1842 & & 231 \\
\hline & & Diapterus rhombeus (Cuvier, 1829) & & 198 \\
\hline & & Eucinostomus gula (Quoy \& Gaimard, 1824) & & 133 \\
\hline & Haemulidae & Pomadasys corvinaeformis (Steindachner, 1868) & 90 & 159 \\
\hline & Sciaenidae & Bairdiella ronchus (Cuvier, 1830) & 138 & 216 \\
\hline & & Isopisthus parvipinnis (Cuvier, 1830) & 120 & 195 \\
\hline & & Larimus breviceps (Cuvier, 1830) & & 152 \\
\hline & & Menticirrhus americanus (Linnaeus, 1758) & 70 & 193 \\
\hline & & Micropogonias furnieri (Desmarest, 1823) & 70 & 243 \\
\hline & & Ophioscion punctatissimus Meek \& Hildebrand, 1925 & & 142 \\
\hline & & Paralonchurus brasiliensis (Steindachner, 1875) & & 58 \\
\hline & & Stellifer rastrifer (Jordan, 1889) & 68 & 133 \\
\hline & & Stellifer stellifer (Bloch, 1790) & 76 & 143 \\
\hline & Ephippidae & Chaetodipterus faber (Broussonet, 1782) & 77 & 490 \\
\hline & Trichiuridae & Trichiurus lepturus Linnaeus, 1758 & 116 & 577 \\
\hline \multirow[t]{6}{*}{ Pleuronectiformes } & Paralichthyidae & Citharichthys spilopterus Gunther, 1862 & 73 & 157 \\
\hline & & Etropus crossotus Jordan \& Gilbert, 1882 & 70 & 121 \\
\hline & Achiridae & Achirus declivis Chabanaud, 1940 & & 92 \\
\hline & & Achirus lineatus (Linnaeus, 1758) & 68 & 135 \\
\hline & & Trinectes paulistanus (Miranda Ribeiro, 1915) & & 80 \\
\hline & Cynoglossidae & Symphurus tessellatus (Quoy \& Gaimard, 1824) & 111 & 172 \\
\hline \multirow[t]{8}{*}{ Tetraodontiformes } & Monacanthidae & Stephanolepis hispidus (Linnaeus, 1766) & & 50 \\
\hline & Tetraodontidae & Lagocephalus laevigatus (Linnaeus, 1766) & 38 & 112 \\
\hline & & Lagocephalus lagocephalus (Linnaeus, 1758) & 49 & 121 \\
\hline & & Sphoeroides greeleyi Gilbert, 1900 & 58 & 114 \\
\hline & & Sphoeroides spengleri (Bloch, 1785) & & 111 \\
\hline & & Sphoeroides testudineus (Linnaeus, 1758) & 193 & 210 \\
\hline & & Sphoeroides tyleri Shipp, 1972 & & 106 \\
\hline & Diodontidae & Chilomycterus spinosus (Linnaeus, 1758) & 105 & 164 \\
\hline
\end{tabular}

Schmidt \& Dias (2012) assumed that at least the Sciaenid fish assemblage has been stable in the Santos Bay for more than 20 years.

Although a single type of gear was used in the Bertioga Channel, this study adds 15 species to the ichthyofauna inventory of Barbanti et al. (2013), whose captures utilized ten different fishing gears over a longer sampling period.

Examples, such as those of the Santos Bay and Bertioga Channel, suggest that the respective sampling sufficiency was not achieved for both areas, as the ichthyofauna has not been adequately sampled. 
Rocha M.L.F. and Dias J.F

Table 3. List of species collected at Continental Shelf, in the 2005 winter and 2006 summer, with respective total maximum (TL max) and minimum (TL min) lengths in millimeters. In case of a single specimen, its length is shown at "TL max" column.

\begin{tabular}{|c|c|c|c|c|}
\hline Order & Family & Species & TL min & TL max \\
\hline Squaliformes & Squalidae & Squalus group megalops/cubensis* & 458 & 477 \\
\hline Squatiniformes & Squatinidae & Squatina argentina (Marini, 1930) & 267 & 557 \\
\hline \multirow[t]{12}{*}{ Rajiformes } & Narcinidae & Narcine brasiliensis (Olfers, 1831) & & 237 \\
\hline & Rhinobatidae & Rhinobatos horkelii, Muller \& Henle 1841 & 250 & 756 \\
\hline & & Rhinobatos percellens (Walbaum, 1792) & 204 & 286 \\
\hline & Rhinobatidae & Zapteryx brevirostris (Muller \& Henle, 1841) & 123 & 493 \\
\hline & Rajidae & Atlantoraja castelnaui (Miranda Ribeiro, 1907) & 256 & 852 \\
\hline & & Atlantoraja cyclophora (Regan, 1903) & 122 & 591 \\
\hline & & Atlantoraja sp. Menni, 1972 & & 454 \\
\hline & & Psammobatis extenta (Garman, 1913) & 87 & 276 \\
\hline & & Psammobatis rutrum Jordan, 1891 & 77 & 283 \\
\hline & & Rioraja agassizii (Muller \& Henle, 1841) & 175 & 560 \\
\hline & Dasyatidae & Dasyatis hypostigma (Santos \& Carvalho, 2004) & & 449 \\
\hline & Myliobatidae & Myliobatis freminvillei Lesueur, 1824 & & $?$ \\
\hline \multirow[t]{3}{*}{ Anguilliformes } & Muraenidae & Gymnothorax ocellatus (Agassiz, 1831) & 279 & 580 \\
\hline & Congridae & Ariosoma opistophthalmus (Ranzini, 1839) & & 293 \\
\hline & & Conger orbignianus Valenciennes, 1837 & & 286 \\
\hline \multirow[t]{10}{*}{ Clupeiformes } & Engraulidae & Anchoa januaria Hildebrand, 1943 & 83 & 168 \\
\hline & & Anchoa spinifer (Valenciennes, 1848) & 128 & 148 \\
\hline & & Anchoviella lepidentostole (Fowler, 1941) & 61 & 129 \\
\hline & & Centegraulis edentulus (Cuvier, 1829) & 87 & 90 \\
\hline & & Engraulis anchoita Hubbs \& Marini, 1935 & 86 & 104 \\
\hline & & Lycengraulis grossidens (Agassiz, 1829) & & 200 \\
\hline & Pristigasteridae & Chirocentrodon bleekerianus (Poey, 1867) & 60 & 113 \\
\hline & & Pellona harroweri (Fowler, 1919) & 34 & 152 \\
\hline & Clupeidae & Harengula clupeola (Cuvier, 1829) & 91 & 186 \\
\hline & & Sardinella brasiliensis (Steindachner, 1879) & 70 & 98 \\
\hline \multirow[t]{2}{*}{ Siluriformes } & Ariidae & Bagre bagre (Linnaeus, 1766) & & 271 \\
\hline & & Genidens genidens (Cuvier, 1829) & & 285 \\
\hline \multirow[t]{2}{*}{ Aulopiformes } & Synodontidae & Saurida brasiliensis Norman, 1935 & 48 & 140 \\
\hline & & Synodus foetens (Linnaeus, 1766) & 85 & 538 \\
\hline Ophidiiformes & Ophidiidae & Raneya brasiliensis (Kaup, 1856) & 194 & 215 \\
\hline \multirow[t]{2}{*}{ Gadiformes } & Phycidae & Urophycis brasiliensis (Kaup, 1858) & 85 & 395 \\
\hline & Merlucciidae & Merluccius hubbsi Marini, 1933 & 82 & 340 \\
\hline Batrachoidiformes & Batrachoididae & Porichthys porosissimus (Cuvier, 1829) & 61 & 332 \\
\hline \multirow[t]{2}{*}{ Lophiiformes } & Lophiidae & Lophius gastrophysus Miranda Ribeiro, 1915 & 83 & 436 \\
\hline & Ogcocephalidae & Ogcocephalus vespertilio (Linnaeus, 1758) & 54 & 142 \\
\hline Zeiformes & Zeidae & Zenopsis conchifer (Lowe, 1852) & & 132 \\
\hline Gasterosteiformes & Fistulariidae & Fistularia petimba Lacepède, 1803 & 295 & 970 \\
\hline \multirow[t]{5}{*}{ Scorpaeniformes } & Dactylopteridae & Dactylopterus volitans (Linnaeus, 1758) & 63 & 310 \\
\hline & Scorpaenidae & Scorpaena brasiliensis Cuvier, 1829 & & 134 \\
\hline & Triglidae & Bellator brachychir (Regan, 1914) & 51 & 71 \\
\hline & & Prionotus nudigula Ginsburg, 1950 & 40 & 212 \\
\hline & & Prionotus punctatus (Bloch, 1793) & 45 & 380 \\
\hline \multirow[t]{14}{*}{ Perciformes } & Acropomatidae & Synagrops spinosus Schultz, 1940 & 60 & 95 \\
\hline & Serranidae & Diplectrum formosum (Linnaeus, 1766) & 90 & 237 \\
\hline & & Diplectrum radiale (Quoy \& Gaimard, 1824) & 82 & 240 \\
\hline & & Dules auriga Cuvier, 1829 & 59 & 163 \\
\hline & Priacanthidae & Priacanthus arenatus Cuvier, 1829 & 66 & 264 \\
\hline & Pomatomidae & Pomatomus saltatrix (Linnaeus, 1766) & & 343 \\
\hline & Carangidae & Caranx crysos (Mitchill, 1815) & & 255 \\
\hline & & Chloroscombrus chrysurus (Linnaeus, 1766) & 41 & 206 \\
\hline & & Decapterus punctatus (Cuvier, 1829) & & 97 \\
\hline & & Oligoplites saliens (Bloch, 1793) & 110 & 478 \\
\hline & & Selene setapinnis (Mitchill, 1815) & 36 & 263 \\
\hline & & Selene vomer (Linnaeus, 1758) & 50 & 236 \\
\hline & & Trachinotus carolinus (Linnaeus, 1766) & 275 & 286 \\
\hline & & Trachurus lathami Nichols, 1920 & 46 & 158 \\
\hline
\end{tabular}


Table 3. Continued.

\begin{tabular}{|c|c|c|c|c|}
\hline Order & Family & Species & TL min & TL max \\
\hline & \multirow[t]{4}{*}{ Gerreidae } & Diapterus rhombeus (Cuvier, 1829) & 115 & 207 \\
\hline & & Eucinostomus argenteus Baird \& Girard, 1855 & 125 & 212 \\
\hline & & Eucinostomus gula (Quoy \& Gaimard, 1824) & & 108 \\
\hline & & Eucinostomus melanopterus (Bleeker, 1863) & 138 & 204 \\
\hline & \multirow[t]{3}{*}{ Haemulidae } & Conodon nobilis (Linnaeus, 1758) & 69 & 236 \\
\hline & & Orthopristis ruber (Cuvier, 1830) & 138 & 296 \\
\hline & & Pomadasys corvinaeformis (Steindachner, 1868) & 77 & 214 \\
\hline & Sparidae & Pagrus pagrus (Linnaeus, 1758) & 40 & 218 \\
\hline & \multirow[t]{15}{*}{ Sciaenidae } & Ctenosciaena gracilicirrhus (Metzelaar, 1919) & 51 & 180 \\
\hline & & Cynoscion acoupa (Lacepède, 1801) & & 196 \\
\hline & & Cynoscion jamaicensis (Vaillant \& Bocourt, 1883) & 70 & 382 \\
\hline & & Cynoscion leiarchus (Cuvier, 1830) & & 242 \\
\hline & & Cynoscion virescens (Cuvier, 1830) & 70 & 435 \\
\hline & & Isopisthus parvipinnis (Cuvier, 1830) & 40 & 160 \\
\hline & & Larimus breviceps (Cuvier, 1830) & 49 & 215 \\
\hline & & Macrodon atricauda (Gunther, 1880) & 50 & 311 \\
\hline & & Menticirrhus americanus (Linnaeus, 1758) & 56 & 292 \\
\hline & & Micropogonias furnieri (Desmarest, 1823) & 189 & 523 \\
\hline & & Nebris microps Cuvier, 1830 & 101 & 169 \\
\hline & & Paralonchurus brasiliensis (Steindachner, 1875) & 50 & 227 \\
\hline & & Stellifer brasiliensis (Shultz, 1945) & 50 & 153 \\
\hline & & Umbrina canosai Berg, 1895 & 115 & 156 \\
\hline & & Umbrina coroides Cuvier, 1830 & 191 & 269 \\
\hline & \multirow[t]{2}{*}{ Mullidae } & Mullus argentinae Hubbs \& Marini, 1933 & 62 & 225 \\
\hline & & Upeneus parvus Poey, 1852 & 54 & 232 \\
\hline & Labridae & Halichoeres sazimai Luiz, Ferreira \& Rocha, 2009 & & 43 \\
\hline & Pinguipedidae & Pseudopercis semifasciata (Cuvier, 1829) & & 280 \\
\hline & Percophidae & Bembrops heterurus (Miranda Ribeiro, 1903) & 88 & 182 \\
\hline & & Percophis brasiliensis Quoy \& Gaimard, 1825 & 143 & 193 \\
\hline & Ephippidae & Chaetodipterus faber (Broussonet, 1782) & 266 & 362 \\
\hline & Sphyraenidae & Sphyraena guachancho Cuvier, 1829 & 98 & 228 \\
\hline & & Sphyraena sp Klein, 1778 & 163 & 180 \\
\hline & & Sphyraena tome Fowler, 1903 & & 186 \\
\hline & Trichiuridae & Trichiurus lepturus Linnaeus, 1758 & 168 & 922 \\
\hline & Stromateidae & Peprilus paru (Linnaeus, 1758) & 34 & 160 \\
\hline \multirow[t]{15}{*}{ Pleuronectiformes } & Paralichthyidae & Citharichthys dinoceros Goode \& Bean, 1886 & & 170 \\
\hline & & Citharichthys macrops Dresel, 1885 & 74 & 177 \\
\hline & & Citharichthys sp. Bleeker, 1872 & 86 & 190 \\
\hline & & Citharichthys spilopterus Gunther, 1862 & & 128 \\
\hline & & Cyclopsetta chittendeni Bean, 1895 & & 250 \\
\hline & & Etropus crossotus Jordan \& Gilbert, 1882 & 97 & 164 \\
\hline & & Etropus longimanus Norman, 1933 & 58 & 126 \\
\hline & & Paralichthys isosceles Jordan, 1891 & 88 & 345 \\
\hline & & Paralichthys patagonicus Jordan, 1889 & 186 & 264 \\
\hline & & Paralichthys sp. Girard, 1858 & 60 & 295 \\
\hline & & Syacium micrurum Ranzani, 1842 & 135 & 196 \\
\hline & & Syacium papillosum (Linnaeus, 1758) & 70 & 246 \\
\hline & & Syacium sp. Ranzani, 1842 & 75 & 298 \\
\hline & & Verecundum rasile Jordan, 1891 & 80 & 265 \\
\hline & Cynoglossidae & Symphurus tessellatus (Quoy \& Gaimard, 1824) & 115 & 200 \\
\hline \multirow[t]{7}{*}{ Tetraodontiformes } & Balistidae & Balistes capriscus Gmelin, 1789 & 186 & 247 \\
\hline & \multirow[t]{2}{*}{ Monacanthidae } & Aluterus monocerus (Linnaeus, 1758) & 298 & 377 \\
\hline & & Stephanolepis hispidus (Linnaeus, 1766) & 40 & 226 \\
\hline & \multirow[t]{2}{*}{ Tetraodontidae } & Lagocephalus laevigatus (Linnaeus, 1766) & 39 & 168 \\
\hline & & Sphoeroides spengleri (Bloch, 1785) & & 91 \\
\hline & \multirow[t]{2}{*}{ Diodontidae } & Chilomycterus spinosus (Linnaeus, 1758) & 35 & 221 \\
\hline & & Diodon hystrix (Linnaeus, 1758) & & 31 \\
\hline
\end{tabular}

*According to Gomes et al., 2010. 
Rocha M.L.F. and Dias J.F.

Table 4. Species added to the fish fauna of the coastal area of São Paulo state, presented in Menezes (2011).

\begin{tabular}{|c|c|c|}
\hline Order & Family & Species \\
\hline Squaliformes & Squalidae & Squalus group megalops/cubensis* \\
\hline \multirow[t]{6}{*}{ Clupeiformes } & Engraulidae & Anchoa filifera (Fowler 1915) \\
\hline & & Anchoa spinifer (Valenciennes 1848) \\
\hline & & Anchoa tricolor (Spix \& Agassiz 1829) \\
\hline & & Anchovia clupeoides (Swainson 1839) \\
\hline & & Anchoviella brevirostris (Gunther 1868) \\
\hline & Pristigasteridae & Chirocentrodon bleekerianus (Poey 1867) \\
\hline \multirow[t]{5}{*}{ Siluriformes } & Ariidae & Aspistor luniscutis (Valenciennes 1840) \\
\hline & & Cathorops spixii (Agassiz 1829) \\
\hline & & Genidens barbus (Lacepède 1803) \\
\hline & & Notarius grandicassis (Valenciennes 1840) \\
\hline & & Potamarius grandoculis (Steindachner 1877) \\
\hline Mugiliformes & Mugilidae & Mugil curvidens Valenciennes 1836 \\
\hline Gasterosteiformes & Fistulariidae & Fistularia tabacaria Linnaeus 1758 \\
\hline Batrachoidiformes & Batrachoididae & Porichthys porosissimus (Cuvier 1829) \\
\hline \multirow[t]{7}{*}{ Perciformes } & Acropomatidae & Synagrops spinosus Schultz 1940 \\
\hline & Haemulidae & Pomadasys corvinaeformis (Steindachner 1868) \\
\hline & Sciaenidae & Cynoscion leiarchus (Cuvier 1830) \\
\hline & & Cynoscion virescens (Cuvier 1830) \\
\hline & & Nebris microps Cuvier 1830 \\
\hline & & Stellifer stellifer (Bloch 1790) \\
\hline & Labridae & Halichoeres sazimai Luiz, Ferreira \& Rocha 2009 \\
\hline Pleuronectiformes & Paralichthyidae & Citharichthys dinoceros Goode \& Bean 1886 \\
\hline & & Cyclopsetta chittendeni Bean 1895 \\
\hline & & Paralichthys isosceles Jordan 1891 \\
\hline & & Syacium micrurum Ranzani 1842 \\
\hline Tetraodontiformes & Tetraodontidae & Lagocephalus lagocephalus (Linnaeus 1758) \\
\hline
\end{tabular}

*According to Gomes et al., 2010.

The results obtained show that part of the region of the SantosSão Vicente estuarine system and adjacent continental shelf present high richness, despite suffering intense pressure from pollution, significant habitat alteration and loss, overfishing, and an important capture of juveniles as bycatch of shrimp fishing. In addition, this study expands the previously reported knowledge of the ichthyofauna in the central coast of São Paulo state.

\section{Acknowledgements}

We are grateful to all people involved in the sampling and sorting of specimens, mainly T. Schmidt, D. Morais, N. Montagner, C. Rondinelli, C. Costa, V. Rodrigues, D. Napolitano, M. Ohkawara, S. Bromberg, and the respective crew of B/Pqs Velliger II, Albacora, and N/Oc Prof. W. Besnard. We thank M. L. Zani-Teixeira for the elaboration of maps. We thank the Oceanographic Institute of the University of São Paulo (USP) for the use of its facilities. Special thanks go to FAPESP (São Paulo Research Foundation) for sponsoring the ECOSAN Thematic Project - (A influência do complexo estuarino da baixada santista sobre o ecossistema da plataforma adjacente) (Proc. N. 03/09932-1) and to CNPq (National Council for Scientific and Technological Development) for the doctorate scholarship granted to M. L. F. Rocha.

\section{References}

ADVANCED SEARCH REPORT (ITIS). World Wide Web electronic publication: http://www.itis.gov/advanced_search.html (last access at $07 / 05 / 2014)$
BARBANTI, B., CAIRES, R. \& MARCENIUK, A.P. 2013. A ictiofauna do Canal de Bertioga, São Paulo, Brasil. Biota Neotrop., 13(1):276-291, doi: 10.1590/S1676-06032013000100027.

CATALOG OF FISHES: http://researcharchive.calacademy.org/ research/Ichthyology/catalog/fishcatmain.asp (last access at 07/05/ 2014).

FACHINNI, B.H. 1995. Ecologia de associações de peixes teleósteos demersais da plataforma continental sudeste do Brasil, de Cabo de São Tomé a Torres $\left(22^{\circ} 04-29^{\circ} 21 \mathrm{~S}\right)$. Tese de doutorado. Instituto Oceanográfico, Universidade de São Paulo, São Paulo.

FIGUEIREDO, J.L. \& MENEZES, N. A. 1978. Manual de peixes marinhos do sudeste do Brasil. II Teleostei (1). Museu de Zoologia Universidade de São Paulo, São Paulo.

FIGUEIREDO, J.L. \& MENEZES, N.A. 1980. Manual de peixes marinhos do sudeste do Brasil. III Teleostei (2). Museu de Zoologia Universidade de São Paulo, São Paulo.

FIGUEIREDO, J.L. \& MENEZES, N.A. 2000. Manual de peixes marinhos do sudeste do Brasil. VI Teleostei (5). Museu de ZoologiaUniversidade de São Paulo, São Paulo.

FISHBASE: http://www.fishbase.org/home.htm (last access at 07/05/2014).

GIANNINI, R. \& PAIVA-FILHO, A.M. 1990. Os Sciaenidae (Teleostei: Perciformes) da baía de Santos (SP), Brasil. Bolm. Inst. Oceanogr., S Paulo, 38(1):69-86, doi: 10.1590/S0373-55241990000100008.

GOMES, U.L., SIGNORI, C.N., GADIG, O.B.F. \& SANTOS, H.R.S. 2010. Guia para identificação de tubarões e raias do Rio de Janeiro. Technical Books, Rio de Janeiro.

GRAÇA-LOPES, R., RODRIGUES, E.S., PUZZI, A., PITA, J.B., COELHO, J.A.P. \& FREITAS, M.L. 1993. Levantamento ictiofaunístico em um ponto fixo na baía de Santos, estado de São Paulo. B. Inst. Pesca, 20:7-20.

HENRIQUES, S., PAIS, M.P., COSTA, M.J. \& CABRAL, H. 2008. Development of a fish based multimetric index to access ecological 
quality of marine habitats: the Marine Fish Comunnity Index. Mar. Pol. Bul., 56:1913-1934, doi: 10.1016/j.marpolbul.2008.07.009.

MARCENIUK, A.P. 2005. Chave para identificação das espécies de bagres marinhos (Siluriformes, Ariidae) da costa brasileira. B. Inst. Pesca, São Paulo, 31(2):89-101.

MARCENIUK, A.P. \& MENEZES, N.A. 2007. Systematics of the family Ariidae (Ostariophysi, Siluriformes), with a redefinition of the genera. Zootaxa, 1416:1-126.

MENEZES, N.A. 2011. Checklist dos peixes marinhos do Estado de São Paulo, Brasil. Biota Neotrop., 11(suppl. 1):33-46, doi: 10.1590/ S1676-06032011000500003.

MENEZES, N.A. \& FIGUEIREDO, J.L. 1980. Manual de peixes marinhos do sudeste do Brasil. IV Teleostei (3). Museu de ZoologiaUniversidade de São Paulo, São Paulo.

MENEZES, N.A. \& FIGUEIREDO, J.L. 1985. Manual de peixes marinhos do sudeste do Brasil. V Teleostei (4). Museu de Zoologia, Universidade de São Paulo, São Paulo.

MENEZES, N.A., BUCKUP, P.A., FIGUEIREDO, J.L. \& MOURA, R.L. 2003. Catálogo das espécies de peixes marinhos do Brasil. São Paulo: EDUSP - Universidade de São Paulo.

MENEZES, N.A., NIRCHIO, M., OLIVEIRA, C. \& SICCHARAMIREZ, R. 2015. Taxonomic review of the species of Mugil (Teleostei: Perciformes: Mugilidae) from the Atlantic South
Caribbean and South America, with integration of morphological, cytogenetic and molecular data. Zootaxa 3918 (1):1-38.

NELSON, J.S. 2006. Fishes of the World. 4a Ed., John Wiley \& Sons, Hoboken.

PAIVA-FILHO, A.M., GIANNINI, R., RIBEIRO NETO, F.B. \& SCHMIEGELOW, J.M.M. 1987. Ictiofauna do Complexo BaíaEstuário de Santos e São Vicente, SP, Brasil. Relat. Int. Inst. Oceanogr., Univ. São Paulo, 17:1-10.

RIBEIRO NETO, F.B. 1989. Estudo da comunidade de peixes da baía de Santos, SP. Dissertação de Mestrado, Instituto Oceanográfico, Universidade de São Paulo, São Paulo.

SCHMIDT, T.C.S \& DIAS, J.F. 2012. Pattern of distribution and environmental influences on the Sciaenidae community of the Southeastern Brazilian coast. Braz. J. Oceanogr., 60(2): 235-245.

VAZZOLER, A.E.A. de M. 1970. Ictiofauna da baía de Santos. I. Sciaenidae (Percoidea, Percomorphi). Bolm. Inst. Oceanogr., S Paulo, 18(1):11-26.

VAZZOLER, G., ZANETTI, E.M., KAWAKAMI, E. \& YAMAGUTI, N. 1982. Teleósteos marinhos coletados entre Cabo Frio $\left(23^{\circ} \mathrm{S}\right)$ e torres $\left(29^{\circ} 21^{\prime} \mathrm{S}\right)$ Programa FAUNEC. Dusenia, Curitiba, 13(3): 127-133.

WORLD REGISTER OF MARINE SPECIES (WorMS). World Wide Web electronic publication: http://www.marinespecies.org (07.05.2014). 